\title{
Grouping and multidimensional organization of respiratory sensations
}

\author{
ANDREW HARVER \\ Dartmouth Medical School, Hanover, New Hampshire \\ JOHN C. BAIRD \\ Dartmouth College, Hanover, New Hampshire \\ and \\ JOAN F. MCGOVERN and J. ANDREW DAUBENSPECK \\ Dartmouth Medical School, Hanover, New Hampshire
}

\begin{abstract}
Agreement has not emerged either on the sensory attributes that underlie psychophysical decisions in respiratory magnitude scaling tasks or on the physical stimuli giving rise to human judgments. Accordingly, sensory attributes of respiratory sensations were explored in the present study by adopting scaling techniques designed to help uncover the organization of sensory experiences elicited by various breathing maneuvers. Physical stimulus characteristics underlying human judgments were also considered. Subjects rated the dissimilarity between 45 pairs of breathing maneuvers on a scale ranging from 0 ("no difference at all") to 10 ("very much difference"). Cluster analysis and multidimensional scaling were employed to uncover the perceived grouping among different types of maneuvers and the relative organization of maneuvers in a Euclidean space. Dissimilarity judgments varied primarily as a function of a single attribute, degree or magnitude of external loading, ranging from an infinite respiratory load to no load at all. This continuum may depend on interactions among pressure or tension, airflow, and lung volume displacement. A secondary attribute may relate to airflow direction (inspiration or expiration).
\end{abstract}

Many types of breathing sensations have been studied, including the sensations of excessive ventilation, tightness in the chest, tracheobronchial irritation, breath holding, and sensations associated with partial or complete occlusion of the airways. Unlike the visual and auditory systems, sensations accompanying breathing do not depend on a single receptor system. In human and animal experiments both direct and indirect evidence is accumulating to suggest that sensory feedback from the upper airways, lung, chest wall, and diaphragm underlie the perception of respiratory stimuli in humans. The multiplicity of receptors that could underlie perceptual judgments reduces the likelihood that the receptor systems subserving respiratory sensations will be easily identified.

Since 1970 (Bakers \& Tenney, 1970), various breathing maneuvers have been examined with Stevens's (1975) magnitude scaling techniques. These maneuvers include producing respiratory efforts against a closed airway and breathing through external devices that impede changes in the rate or volume of breathing. From these types of investigations, a cohesive set of exponents has been gathered both for different scaling tasks and for performance on these tasks by various subpopulations. For ex-

Supported by Training Grant HL07449 and by Grant HL29068 from the National Heart, Lung, and Blood Institute, National Institutes of Health. Address requests for reprints to Andrew Harver, Department of Psychology, SUNY Stony Brook, Stony Brook, NY 11794-2500. ample, exponents for static respiratory efforts produced against a closed airway in young adults average about 1.7 (e.g., Harver \& Kotses, 1987; Tack, Altose, \& Cherniack, 1983). Exponents for the perceived magnitude of resistive loads added to inspiration (i.e., airway obstructions that reduce the rate of airflow) in patients with obstructive lung disease average about .5 (e.g., Gottfried, Redline, \& Altose, 1985; Mahler et al., 1987); those in young adults are about .8 (e.g., Ward \& Stubbing, 1985).

Despite the reliability of these and related findings, agreement on the receptor mechanisms giving rise to human judgments on respiratory scaling tasks, or on the sensory attributes that underlie psychophysical decisions, has not emerged. Physical stimulus characteristics hypothesized to mediate respiratory sensations include tension resulting from contracting respiratory muscles, displacement from lung volume feedback, and effort from collateral motor command signals (e.g., Gottfried, Leech, Dimarco, Zaccardelli, \& Altose, 1984; Killian \& Campbell, 1985; Tack et al., 1983). However, the psychophysical relations between stimulus and sensation in breathing remain largely unspecified.

The fundamental issue to be addressed here is how best to develop a vocabulary of attributes describing the respiratory parameters or dimensions subjects attend to when they participate in magnitude scaling tasks. In most psychophysical experiments, agreement on the nature of the relationship between the attribute and the stimulus has 
evolved. For example, in the case of hearing, it is generally accepted that the attributes of loudness and pitch are closely linked with the physical properties of sound intensity and frequency. In other modalities, there are notable exceptions. In the case of pain, identification of attributes and stimuli has only recently been the topic of systematic investigation (Clark, Carroll, Yang, \& Janal, 1986). In this regard, the status of research on respiration is more closely analogous to that on pain than to that on hearing. As with pain, respiration may vary along reliable dimensions that are not intuitively obvious (Altose, 1985; Clark, 1984).

One approach to the problem of achieving a psychophysical characterization of breathing is to examine variations in stimulus variables that affect the perception of a single sensation. For example, Killian and his colleagues (Killian, Bucens, \& Campbell, 1982; Killian, Mahutte, \& Campbell, 1981) examined the effects of alterations in the breathing pattern on the ability of subjects to scale flow-resistive and elastic loads added to breathing. Fixing tidal volume (to 1 liter) during the addition of elastic loads and flow rates (to 1 liter/sec) during the addition of resistive loads caused an increase in the exponents for added loads as compared with when subjects freely adopted their tidal volume and flow rate (Killian et al., 1981). The perceived magnitude of both resistive and elastic loads also increased as inspiratory duration increased (Killian et al., 1982). These researchers concluded that perceived magnitude of external loads depends on such factors as tidal volume or flow rate, or factors related to the degree and duration of inspiratory muscle force, and is related only indirectly to load type. Such a conclusion indicates that certain stimulus variables affect perceptual judgments, but they fail to provide insight to the sensory experiences subjects attend to when they participate in magnitude scaling tasks. For example, alterations in exponents when the breathing pattern is constrained to a certain pattern may be confounded by variations in the difficulty of constraining respiration to a particular pattern.

It is difficult to see how magnitude scaling approaches can provide the evidence that would favor the attributes of sensory experiences over any other. Magnitude scaling requires that the subject attend to a single sensory aspect of breathing closely specified by the experimenter's instructions (e.g., Killian, Gandevia, Summers, \& Campbell, 1984). An alternative set of methods designed to uncover possible parameters or dimensions of experience are those that rely on clustering and multidimensional scaling algorithms. Such techniques recently have proven valuable in the study of pain (Clark et al., 1986). With these methods, the observer represents his or her perceptual world through ratings of similarity or dissimilarity among stimulus objects (Baird \& Noma, 1978). Analysis of these ratings produces a configuration of emergent properties of different stimuli. Such a configuration may offer a firmer basis for theory construction, for instance, in interpreting the relative sizes of exponents under different experimental conditions (e.g., Baird, 1970; Stevens, 1975; Teghtsoonian, 1971).
The present study employed cluster analysis and multidimensional scaling (MDS) to help uncover the organization of sensory experiences surrounding respiratory sensations elicited by various breathing maneuvers. To accomplish this, we examined the most common maneuvers reported in the respiratory literature (see Zechman \& Wiley, 1986)-breathing against a closed shutter, breathing through resistive and elastic loads, and varying the depth of inspiration. Static maneuvers-that is, those performed against a closed shutter-impose an infinite load on the respiratory system; flow does not occur and therefore volume cannot change regardless of the applied pressure. Both flow and volume change while the subject is breathing through resistive and elastic loads, but only because the applied pressure increases in proportion to the applied impediment to breathing (respectively, mesh screens and closed containers). From a clinical standpoint, resistive loads mimic the type of flow limitation experienced by patients with obstructive lung disease (e.g., chronic bronchitis), whereas elastic loads mimic the type of volume restriction experienced by patients with interstitial lung disease (e.g., pulmonary fibrosis). Two levels each of resistance and elastance were examined because variations in the levels of these loads might affect perceptual judgments differentially.

In addition, subjects experienced some maneuvers in both inspiratory and expiratory directions. A priori, there is little reason to expect airflow direction to affect the perceptual organization of respiratory sensations. For example, exponents for scaling resistive loads added to inspiration are comparable to those added to expiration (Muza, McDonald, \& Zechman, 1984); exponents for scaling static inspiratory forces are comparable to those for expiratory static forces (Harver \& Kotses, 1987).

Two separate analyses were conducted on ratings of dissimilarity obtained between pairs of breathing maneuvers. The first, cluster analysis, produces a hierarchical tree showing each individual maneuver at the top and the linkings among all maneuvers in a nested arrangement defined by perceived similarity. The second, MDS, creates a "group stimulus space"- a map, where each point corresponds to one of the maneuvers. The relative locations of points on the map depend on the relationship between all possible pairs of stimuli (the greater the dissimilarity between maneuvers, the greater their interpoint distance in the space). The ordering of the stimuli between two extreme points then allows us to draw inferences about the nature of the dimension (i.e., the attribute) along which ratings vary. We found that the MDS space for the common maneuvers we examined was described adequately by two dimensions that we labeled and then attempted to trace to underlying, physical stimulus characteristics.

\section{METHOD}

\section{Subjects}

The participants were 14 Dartmouth College students $(6$ male and 8 female), ranging in age between 18 and 20 years. All students received extra course credit in introductory psychology for their participation. 


\section{Apparatus}

The breathing circuit, shown in Figure 1, was constructed of spiral plastic tubing (1.5" i.d.) attached to both inspiratory and expiratory ports of a Daniels valve. Pressure near the mouth was recorded by a pressure transducer (Vacumetrics No. $4500, \pm 100 \mathrm{cmH}_{2} \mathrm{O}$ ) and carrier demodulator (No. 4512-100). Airflow was monitored continuously by a Hans Rudolph pneumotachometer and heater (Model 3813). Both the pressure and airflow signals were displayed on separate channels of a Honeywell thermal recorder (Model 1400). A T-shape stopcock (Collins No. 21043) was interposed between the pneumotachometer and the Daniels valve and was used to occlude the circuit manually during the static maneuvers. The resistive loads ( 22 and $43 \mathrm{cmH}_{2} \mathrm{O} /$ liter/sec) were constructed from tubing, $2 \mathrm{~cm}$ in diameter, and nylon mesh, and were linear to a flow rate of 1 liter/sec. The elastic loads $\left(45\right.$ and $65 \mathrm{cmH}_{2} \mathrm{O} /$ liter) consisted of airtight, glass containers. Both the resistive and elastic loads were placed behind the subject and were attached to the spiral plastic tubing with rigid rubber tubing and a series of T-shape stopcocks and low-turbulence three-way $Y$ valves (Collins No. 21041). The arrangement of the stopcocks and $Y$ valves allowed the experimenter to add loads selectively either to inspiration (resistive or elastic) or to expiration (resistive only). The average circuit resistance was less than $1 \mathrm{cmH}_{2} \mathrm{O} /$ liter/sec; the total dead space was less than $300 \mathrm{ml}$.

\section{Procedure}

Each student participated in one 60-min experimental session. The subject was seated in a dental chair, a nose clamp was applied, and instruction was given to breathe "at ... normal depth and rate" on the breathing circuit for $2 \mathrm{~min}$. Next, the subject experienced sensations elicited by nine different breathing maneuvers. Some maneuvers required the subject to breathe against a closed airway, or to inspire about twice as much as usual; others required the subject to breathe against resistive or elastic loads. The following conditions were examined (see Table 1): static inspiratory and expiratory efforts (i.e., with the airway occluded) produced at the end of a normal breath, a large unimpeded inspiration, high and low inspiratory resistances, high and low expiratory resistances, and high and low inspiratory elastances. Each condition was preceded by a verbal warning delivered during the phase of respiration preceding the target phase, and was encountered once during a single inspiration or expiration. Breathing patterns during this initial period, and during the main portion of the experiment, were not constrained; subjects freely adopted their own frequency and volume of breathing. Data were not recorded from records made during the initial period, which served only to familiarize the subject with the breathing circuit as well as with the type and range of conditions.

Following this, the subjects rated the subjective dissimilarity between pairs of sensations, elicited by the various breathing maneu-

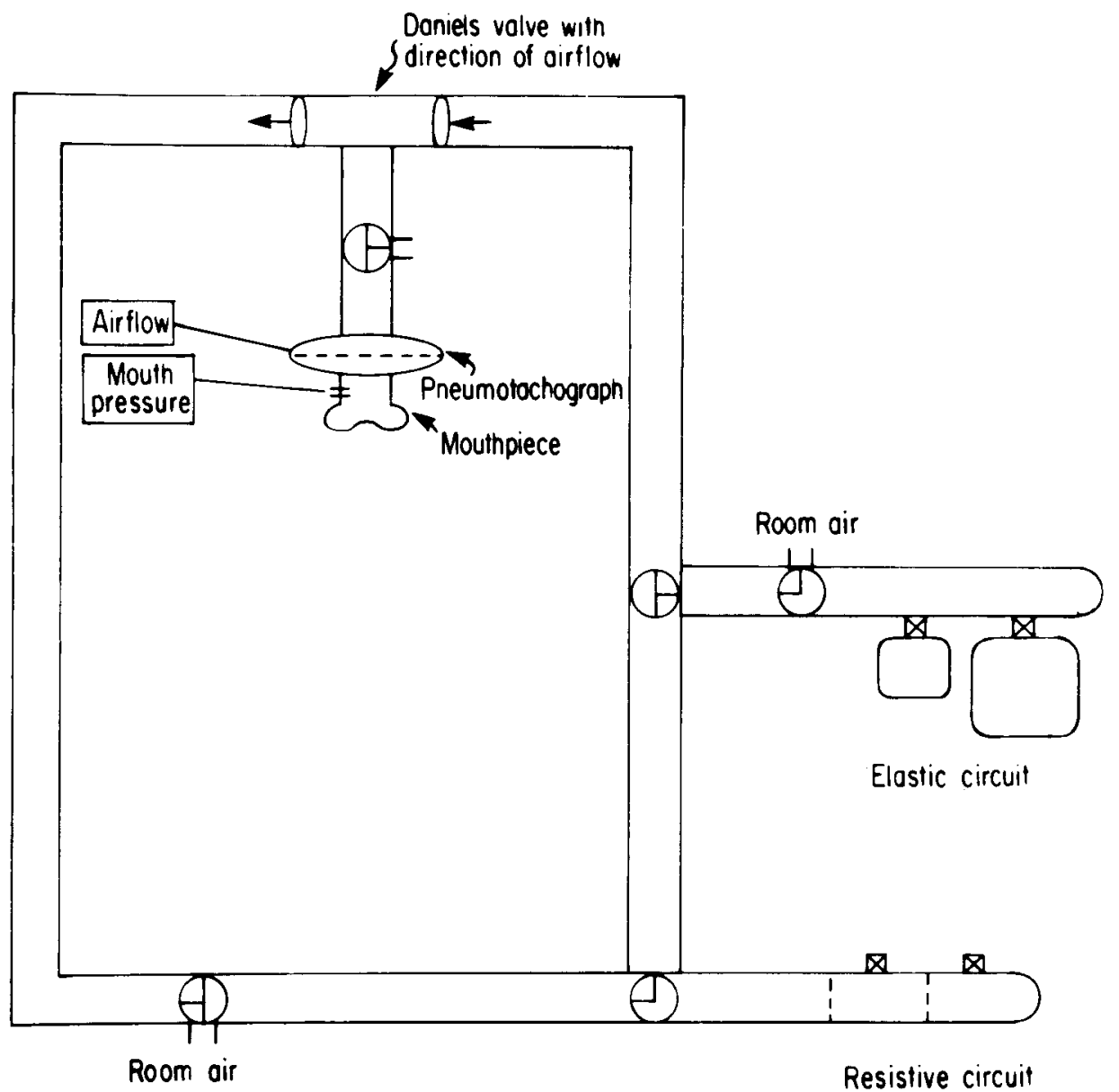

Figure 1. Schematic of breathing apparatus. 
Table 1

Breathing Maneuvers

\begin{tabular}{|c|c|}
\hline Condition & Maneuver \\
\hline Static inspiration & Inspiration against a closed airway from FRC (INS) \\
\hline Static expiration & Expiration against a closed airway from FRC (EXS) \\
\hline Unimpeded inspiration & A breath about twice as large as normal (INN) \\
\hline $\begin{array}{l}\text { Inspiratory resistance } \\
22 \mathrm{cmH}_{2} \mathrm{O} / \text { liter/sec } \\
43 \mathrm{cmH}_{2} \mathrm{O} / \text { liter/sec }\end{array}$ & $\begin{array}{l}\text { Inspiration through a resistance of low intensity (IRL) } \\
\text { Inspiration through a resistance of high intensity (IRH) }\end{array}$ \\
\hline $\begin{array}{l}\text { Expiratory resistance } \\
22 \mathrm{cmH}_{2} \mathrm{O} / \text { liter/sec } \\
43 \mathrm{cmH}_{2} \mathrm{O} / \text { liter/sec }\end{array}$ & $\begin{array}{l}\text { Expiration through a resistance of low intensity (ERL) } \\
\text { Expiration through a resistance of high intensity (ERH) }\end{array}$ \\
\hline $\begin{array}{l}\text { Inspiratory elastance } \\
45 \mathrm{cmH}_{2} \mathrm{O} / \text { liter } \\
65 \mathrm{cmH}_{2} \mathrm{O} / \text { liter }\end{array}$ & $\begin{array}{l}\text { Inspiration through an elastance of low intensity (IEL) } \\
\text { Inspiration through an elastance of high intensity (IEH) }\end{array}$ \\
\hline
\end{tabular}

vers, on a scale ranging between 0 ("'no difference between stimuli") and 10 ("very much difference"). Each subject rated a total of 45 pairs, the lower triangle of the $9 \times 9$ matrix of all possible pairs of maneuvers, including the diagonal (i.e., each maneuver paired with itself). Two to three normal breaths separated each maneuver within pairs; the order within pairs was counterbalanced for all subjects. Eight to 10 normal breaths separated each pair, and the sequence was randomized for all subjects. The subjects were told only that a manipulation of the breathing circuit would occur on the "next breath" except for the large inspiration, which necessarily was introduced as a "big breath" during the preceding expiration. After the second maneuver in each pair, the subject recorded a dissimilarity judgment on a sheet of paper.

Subjects were instructed to make the same magnitude of effort on all maneuvers and to rate only the sensations elicited by the various maneuvers. In this way, subjects likely approached each maneuver in a consistent fashion, although completion of the maneuvers may have required differential amounts of subjective effort.

\section{Data Reduction}

Mean dissimilarity judgments were computed for each unique pair of maneuvers and were analyzed using both cluster analysis and multidimensional scaling (specifically, McGee's, 1966, analysis of "elastic" distances).

\section{Cluster Analysis}

A hierarchical grouping of maneuvers (Johnson, 1967) was obtained by using a complete linkage cluster analysis of the mean dissimilarity ratings for each pair. In this analysis, each level of clustering is determined by comparing all possible distances between individual maneuvers as well as between groupings formed earlier in the clustering process. In complete linkage, the distance from a single stimulus and a cluster is taken as the distance between it and the farthest stimulus in the cluster. This makes it comparatively difficult for an outside stimulus to be added to an existing cluster. A hierarchical tree is thereby constructed showing individual maneuvers at the top of a branch and showing how they cluster as one moves down the tree toward the trunk, which eventually contains all the maneuvers (see also Baird \& Noma, 1978, chap. 11).

\section{Multidimensional Scaling}

In our application, the method solves for an optimal spatial configuration of maneuvers based on their rank order of dissimilarity. We treat mean dissimilarity ratings as distances in a Euclidean space. The scaling procedure attempts to place stimuli in this space along a minimum number of interpretable dimensions in order to best satisfy all the pair distances existing in the data matrix. For our purposes, both one- and two-dimensional solutions were obtained and are presented in the following results section (see also Baird \& Noma, 1978, chap. 10).

\section{RESULTS}

Mean dissimilarity judgments ranged between 0.50 (dissimilarity between two unimpeded inspirations) and 9.14 (dissimilarity between an unimpeded inspiration and an expiration against a closed airway). Mean judgments for all unique stimulus pairs are given in Table 2.

The cluster analysis grouped maneuvers based on the mean estimates of dissimilarity. The first level of grouping indicated that the high and low inspiratory elastances were the most homogeneous elements within the set of maneuvers examined. This observation is represented as the highest level in Figure 2 where these two maneuvers are linked. Subsequent clusters were formed in a hierarchical fashion through combinations of similar elements, or groups of elements, until the set was exhausted (bottom of the tree). Note that as clustering proceeded, one broad property reflected in the groupings involved qualities or kinds of external loading; near the bottom of the tree, for example, the unimpeded inspiration (no load) is separated from both static respirations (infinite loads) and added resistances and elastances (partial loads).

The one- and two-dimensional MDS analyses gave reasonable solutions. The one-dimensional solution for the dissimilarity judgments between pairs of breathing maneuvers is shown in Figure 3. The arrangement of points along the horizontal axis, ranging from complete loading of the system (static respirations) to no external loading at all (unimpeded inspiration), indicate that the perceived degree or magnitude of respiratory loading affected dissimilarity judgments. This alignment and the preceding cluster solution are shown together in Figure 4, achieved by superimposing the first six levels of the cluster analysis directly on the one-dimensional solution. This conjoint display highlights the observations that organization of the breathing maneuvers in the space is related to the degree or magnitude of impedance opposing respiration.

The two-dimensional solution is shown in Figure 5. As before, the location of points along the horizontal axis 
Table 2

Matrix of Mean Dissimilarity Judgments

\begin{tabular}{ccccccccccccc}
\hline $\begin{array}{c}\text { Static } \\
\text { inspiration }\end{array}$ & $\begin{array}{c}\text { Static } \\
\text { expiration }\end{array}$ & $\begin{array}{c}\text { Unimpeded } \\
\text { inspiration }\end{array}$ & $\begin{array}{c}\text { Inspiratory } \\
\text { resistance } \\
\text { (high) }\end{array}$ & $\begin{array}{c}\text { Inspiratory } \\
\text { resistance } \\
\text { (low) }\end{array}$ & $\begin{array}{c}\text { Expiratory } \\
\text { resistance } \\
\text { (high) }\end{array}$ & $\begin{array}{c}\text { Expiratory } \\
\text { resistance } \\
\text { (low) }\end{array}$ & $\begin{array}{c}\text { Inspiratory } \\
\text { elastance } \\
\text { (high) }\end{array}$ & $\begin{array}{c}\text { Inspiratory } \\
\text { elastance } \\
\text { (low) }\end{array}$ \\
\hline
\end{tabular}

Static

inspiration $\quad 1.21 \quad 1.53$

Static

expiration

Unimpeded

inspiration

Inspiratory

$\begin{array}{lllllllll}\text { resistance (high) } & 4.07 & 2.89 & 3.93 & 3.29 & 7.07 & 2.59 & 1.64 & 1.91\end{array}$

Inspiratory

$\begin{array}{lllllllllll}\text { resistance (low) } & 5.43 & 2.68 & 5.14 & 2.51 & 7.21 & 2.49 & 2.71 & 2.73 & 1.07 & 1.33\end{array}$

Expiratory

$\begin{array}{lllllllllllll}\text { resistance (high) } & 5.36 & 2.79 & 3.71 & 2.23 & 6.50 & 2.65 & 3.50 & 2.88 & 4.93 & 3.05 & 0.57 & 0.76\end{array}$

Expiratory

resistance (low)

Inspiratory

elastance (high)

$\begin{array}{llll}2.57 & 2.93 & 0.79 & 1.63\end{array}$

Inspiratory

elastance (low)

5.792 .55

5.142 .68

$6.29 \quad 2.8$

$5.14 \quad 1.88$

$\begin{array}{llll}4.00 & 2.63 & 2.00 & 2.48\end{array}$

$2.14 \quad 1.88$

$\begin{array}{llllll}4.43 & 2.71 & 4.64 & 3.41 & 7.43 & 2.93\end{array}$

$2.14 \quad 2.18$

$3.86 \quad 2.63$

$\begin{array}{llll}4.86 & 2.71 & 5.07 & 2.92\end{array}$

$1.57 \quad 1.34$

$\begin{array}{llllllll}4.29 & 2.84 & 4.64 & 3.10 & 6.43 & 3.37 & 2.14 & 1.56\end{array}$

$\begin{array}{lll}2.57 & 2.38\end{array}$

$\begin{array}{llllll}4.93 & 2.73 & 5.07 & 2.76 & 1.64 & 1.91\end{array}$

$1.57 \quad 2.31$

Note $-N=14$.

seems to indicate an impedance dimension. The organization of points along the vertical axis presents ambiguous extremes by which to identify a dimension. Possibly, this second dimension is airflow direction (inspiration or expiration).

Finally, we consider the relationship between mean ratings of dissimilarity between pairs of breathing maneuvers and the average levels of peak tension (mouth pressure) produced on each task. Mean levels of peak mouth pressure associated with each breathing maneuver are given in Table 3 . These values were compared to the coor-

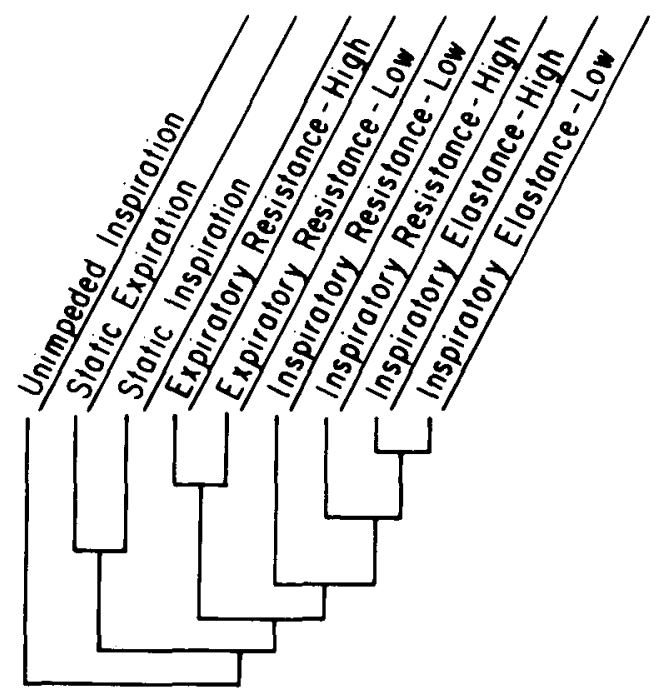

Figure 2. Hierarchical clustering solution for dissimilarity judgments obtained between pairs of breathing sensations. See text. dinate position of each stimulus in the one-dimensional solution (Figure 3). The location of each stimulus and the associated peak pressure were reliably related $(r=0.70$, $p<.05$ ).

\section{DISCUSSION}

The results of two analyses of dissimilarity judgments of breathing maneuvers were complementary. Both procedures provided evidence that individuals organize sensations elicited by these maneuvers primarily along a single continuum, that is, in terms of the degree or magnitude of impedance opposing respiration. This is consistent with what is known about how the respiratory system operates. For example, receptors in the extrathoracic airways respond to changes in both negative (i.e., inspiratory) and positive (i.e., expiratory) pressures (Sant'Ambrogio, 1982). The major implication of our results concerns the nature of the sensory attribute used by subjects in making judgments in respiratory tasks: Although in the past a variety of instructions have been used in such tasks (e.g., Altose, Leitner, \& Cherniack, 1985, instructed subjects to judge resistance rather than force used to overcome resistive loads; Killian et al., 1984, instructed subjects to judge, separately, tension and effort during elastic loading), subjects may experience variations in respiratory sensations along a single perceptual dimension. This dimension (i.e., degree or magnitude of external loading) may provide the framework for organizing various breathing sensations on the basis only of the load opposing the respiratory system. 


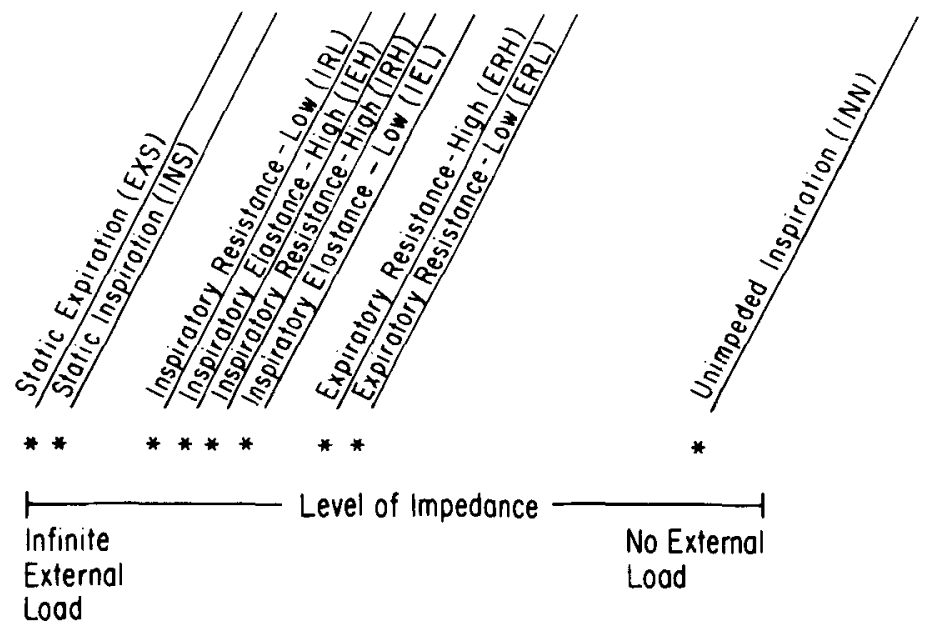

Figure 3. One-dimensional MDS outcome for dissimilarity judgments obtained between pairs of breathing sensations. Each point along the single, horizontal axis is associated with a fixed, scaled location.

On the basis of the MDS solution, we can speculate about the physical stimulus characteristics that underlie the perceptual organization of sensation. For example, the maneuvers depicted in the MDS space are related closely to actual levels of pressure developed against the various loads. The alignment of breathing maneuvers within the dimension might depend, therefore, on signals related to pressure. Such a conclusion is consistent with experimental findings that use standard psychophysical approaches in which the perceived magnitude of respiratory sensations is related directly to pressure developed while overcoming static, resistive, and elastic loads (e.g., Altose, Dimarco, Gottfried, \& Strohl, 1982; Gottfried et al., 1984; Killian et al., 1981, 1982; Muza et al., 1984).

On the other hand, the alignment of breathing maneuvers within the dimension covaries not only with level of pressure (ranging from high to low), but also with level of airflow (ranging from none to high). The alignment of breathing maneuvers within the dimension might depend, therefore, on signals connected only with airflow. Such a hypothesis is consistent with recent evidence of flow-dependent (i.e., temperature sensitive) extrathoracic receptors (Sant'Ambrogio, Mathew, Fisher, \& Sant'Ambrogio, 1983; Sant'Ambrogio, Mathew, Sant'Ambrogio, \& Fisher, 1985).

An indirect argument favoring airflow signals over pressure or muscle tension signals in the perceptual organization of respiratory sensations can be developed by noting inconsistent covariations between the location of each maneuver in the one-dimensional array and the associated level of pressure. The major divergence involves the comparison of the location of a static expiration (Figure 3) with the rank order of pressure produced during the maneuver (Table 3). This discrepancy appears less marked, however, when the adequate stimulus is assumed to be airflow. In that case, a static expiration is predictably adjacent to the other no-flow task, a static inspiration (Figure 3). Unfortunately, actual airflow levels were not determined, so the relationship between airflow and the position of each breathing maneuver in the onedimensional solution could not be compared with that obtained between pressure and each maneuver.

Pressure or airflow could govern dissimilarity ratings, but the exact physical stimuli underlying these judgments cannot be determined unequivocally from the present data. The alignment of maneuvers along the impedance dimension follows not only pressure and airflow but also displacement (ranging from none to high) and, conceivably, effort (ranging from high to none). Thus, an argument could be made that any one-but only one-of the sensory signals previously hypothesized to mediate the magnitude of respiratory sensations (i.e., tension resulting from contracting respiratory muscles, displacement from lung volume feedback, and effort from collateral motor command signals) or airflow influences dissimilarity ratings.

Alternatively, the one-dimensional solution may depend on complex interactions among physical stimuli at any point along the "infinite external load-no external load"

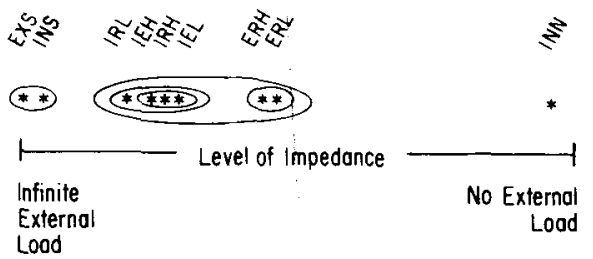

Figure 4. Superimposition of the first six levets of the cluster anglysis on the one-dimensional MDS outcome. (Abbreviations as in Figure 3.) 


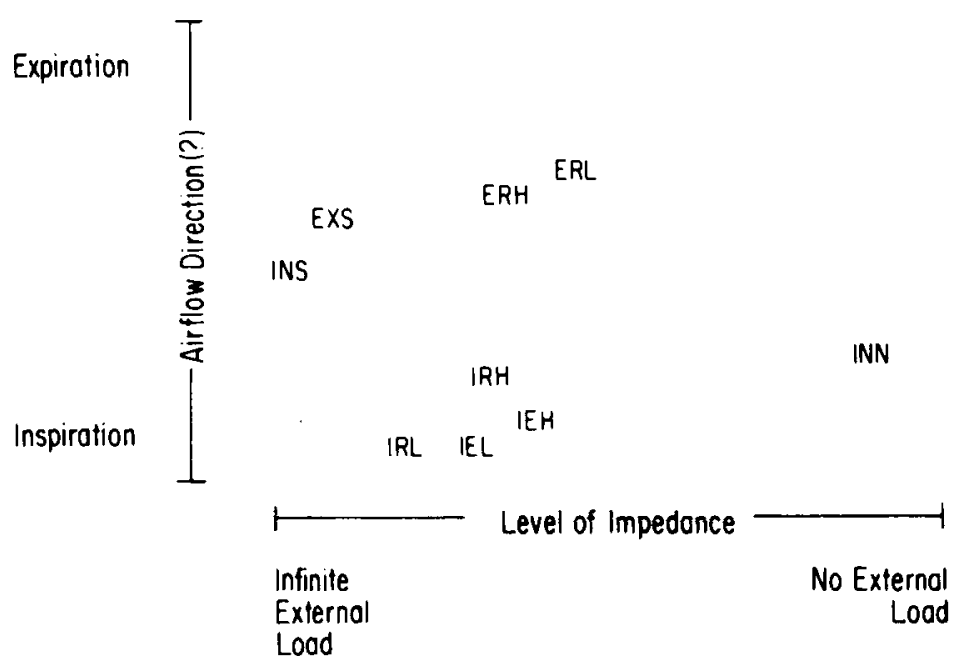

Figure 5. Two-dimensional MDS outcome for dissimilarity judgments obtained between pairs of breathing sensations. (Abbreviations as in Figure 3.)

dimension. The mediation of dissimilarity ratings may arise, for example, from integration or gating of pressure, airflow, and displacement; the role of effort may need to be considered in this context as well. The possibility of perception arising from interactions among these variables for various breathing maneuvers has been discussed (e.g., Campbell, Bennett, \& Rubenstein, 1963; Tack et al., 1983; Teghtsoonian \& Teghtsoonian, 1982). For example, physical stimuli such as flow rates and tidal volume affect exponents for added resistive loads (Killian et al., 1982). Also, this notion of a single, dynamic continuum can incorporate formulations based on the "length:tension appropriateness" hypothesis (Campbell \& Howell, 1963). According to that hypothesis, perception of threshold respiratory loads depends on mismatches between the displacement (volume or length) or rate of change in displacement achieved for the pressure (or tension) exerted. The position of breathing maneuvers in the one-dimensional solution provides an empirical context in which to consider the possible dependence between volume and tension signals.

Table 3

Rank Order of Average Levels of Peak Pressure Generated for Each Condition

\begin{tabular}{lrc}
\hline & \multicolumn{2}{c}{ Mouth Pressure } \\
\cline { 2 - 3 } \multicolumn{1}{c}{ Condition } & \multicolumn{1}{c}{$M$} & $S D$ \\
\hline Static inspiration (INS) & 10.96 & 4.63 \\
Inspiratory elastance, low (IEL) & 9.59 & 4.05 \\
Inspiratory elastance, high (IEH) & 9.52 & 3.15 \\
Inspiratory resistance, high (IRH) & 9.05 & 3.46 \\
Inspiratory resistance, low (IRL) & 8.22 & 3.15 \\
Static expiration (EXS) & 7.63 & 2.73 \\
Expiratory resistance, high (ERH) & 5.85 & 2.12 \\
Unimpeded inspiration (INN) & 5.56 & 3.37 \\
Expiratory resistance, low (ERL) & 5.21 & 2.02 \\
\hline
\end{tabular}

Note-Values given in $\mathrm{cmH}_{2} \mathrm{O} . \mathrm{N}=14$.
A single continuum of respiratory sensation may also help us understand perceptual differences among subpopulations. For example, differences in power function exponents exist between young and old subjects, but are affected by the type of breathing maneuver: exponents for static tasks are the same in young and old; mechanical loads are greater in younger subjects; and production of inspiratory volumes are greater in older subjects (Tack, Altose, \& Chemiack, 1981, 1982, 1983). These findings may be due to differential integration or gating of signals along an impedance dimension in young and old subjects.

The present findings agree with recent arguments based on magnitude estimation indicating a central role of impedance in respiratory sensation. However, our discussions do not address effects of inspiratory or expiratory duration on dissimilarity ratings. Airflow durations ranging between 2 and 6 sec have been shown to affect exponents of power functions for various kinds of respiratory scaling tasks (e.g., Killian et al., 1982). However, other experimenters have suggested that magnitude estimations are not affected by consistently short inspiratory and expiratory durations in the range of 2 to $3 \mathrm{sec}$ (Muza et al., 1984). Our discussions also do not address arguments that breathlessness is a sensation of the efferent (i.e., central) motor effort required of the respiratory muscles (Killian \& Campbell, 1985). The implications of results from respiratory scaling tasks for understanding the sensation of breathlessness remain unclear. For example, exponents for resistive loads in patients with obstructive airway disease are independent of clinical ratings of breathlessness (Mahler et al., 1987).

Although the two-dimensional MDS outcome suggests that a second factor affects the organization of breathing maneuvers, the stimulus, as well as the meaning, underlying this second dimension is ambiguous (Figure 5). It may reflect airflow direction. If the location of static in- 
spiration (INS) were somewhat different, such a conclusion could be quite satisfactory. In other words, airflow direction may fundamentally influence perceptual experiences associated with respiration even though exponents obtained for various inspiratory and expiratory scaling tasks are comparable. On the other hand, given the uncertainty surrounding this interpretation, alternatives can be entertained. For example, the dimension might reflect "novelty of task" ranging from "most unusual" to "least unusual." Further research will be needed to resolve this matter.

\section{REFERENCES}

Altose, M. D. (1985). Assessment and management of breathlessness. Chest, 88(Suppl.), 77-82.

Altose, M. D., Dimarco, A. F., Gottfried, S. B., \& Strohl, K. P. (1982). The sensation of respiratory muscle force. American Review of Respiratory Disease, 126, 807-811.

Altose, M. D., Leitner, J., \& Cherniack, N. S. (1985). Effects of age and respiratory efforts on the perception of resistive ventilatory loads. Joumal of Gerontology, 40, 147-153.

BAIRD, J. C. (1970). A cognitive theory of psychophysics. II. Fechner's law and Stevens' law. Scandinavian Joumal of Psychology, 11, 89-102.

BAIRD, J. C., \& Noma, E. (1978). Fundamentals of scaling and psychophysics. New York: Wiley.

Bakers, J. H. C. M., \& Tenney, S. M. (1970). The perception of some sensations associated with breathing. Respiratory Physiology, 10, 85-92.

Campbell, E. J. M., Bennett, E. D., Rubenstein, D. (1963). The ability to distinguish between added elastic and resistive loads to breathing. Clinical Science, 24, 201-207.

Camprell, E. J. M., Howell, J. B. L. (1963). The sensation of breathlessness. British Medical Bulletin, 19, 36-40.

Clark, W. C. (1984). Application of multidimensional scaling to problems in experimental and clinical pain. In B. Brown (Ed.), Pain measurement in man: Neurophysiological correlates of pain (pp. 349369). Amsterdam: Elsevier.

Clark, W. C., Carroll, J. D., Yang, J. C., Janal, M. N. (1986). Multidimensional scaling reveals two dimensions of thermal pain. Journal of Experimental Psychology: Human Perception \& Performance, 12, 103-107.

GotTFried, S. B., Leech, I., Dimarco, A. F., Zaccardelli, W., \& Altose, M. D. (1984). Sensation of respiratory force following low cervical spinal cord transection. Joumal of Applied Physiology, 57, 989-994.

Gottfried, S. B., Redline, S., \& Altose, M. D. (1985). Respiratory sensation in chronic obstructive pulmonary disease. American Review of Respiratory Disease, 132, 954-959.

HARVER, A., KotSEs, H. (1987). Perception of static respiratory forces in young and old subjects. Perception \& Psychophysics, 41, 449-454.

JoHnson, S. C. (1967). Hierarchical clustering schemes. Psychometrika, $32,241-254$

Killian, K. J., Bucens, D. D., \& Campbell, E. J. M. (1982). Effect of breathing patterns on the perceived magnitude of added loads to breathing. Journal of Applied Physiology, 52, 578-584.

Killan, K. J., a Campbell, E. J. M. (1985). Dyspnea. In C. Roussos \& P. T. Macklem (Eds.), The thorax (part B, pp. 787-828). New York: Marcel Dekker.

Kiluan, K. J., Gandevia, S. C., Summers, E., Campbell, E. J. M. (1984). Effect of increased lung volume on perception of breathlessness, effort, and tension. Journal of Applied Physiology, 57, 686-691.

Killan, K. J., Mahutte, C. K., Campbell, E. J. M. (1981). Magnitude scaling of externally added loads to breathing. American Review of Respiratory Disease, 123, 12-15.

Mahler, D. A., Rosiello, R. A., Harver, A., Lentine, T., McGovern, J. F., \& Daubenspeck, J. A. (1987). Comparison of clinical dyspnea ratings and psychophysical measurements of respiratory sensation in obstructive airway disease. American Review of Respiratory Disease, 135, 1229-1233.

MCGEE, V. E. (1966). The multidimensional analysis of "elastic" distances. British Joumal of Mathematical \& Statistical Psychology, 19, 181-196.

Muza, S. R., McDonald, S., \& Zechman, F. W. (1984). Comparison of subjects' perception of inspiratory and expiratory resistance. Joumal of Applied Physiology, 56, 211-216.

SANT'AMBrogio, G. (1982). Information arising from the tracheobronchial tree of mammals. Physiological Reviews, 62, 531-569.

Sant'Ambrogio, G., Mathew, O. P., Fisher, J. T., Sant'ambroGIO, F. B. (1983). Laryngeal receptors responding to transmural pressure, airflow, and local muscle activity. Respiration Physiology, 54, 317-330.

Sant'Ambrogio, G., Mathew, O. P., Sant'Ambrogio, F. B., Fisher, J. T. (1985). Laryngeal cold receptors. Respiration Physiology, 59, 35-44.

Stevens, S. S. (1975). Psychophysics: Introduction to its perceptual, neural, and social prospects. New York: Wiley.

TACK, M., Altose, M. D., \& Cherniack, N. S. (1981). Effect of aging on respiratory sensations produced by elastic loads. Journal of Applied Physiology, 50, 844-850.

TACK, M., Altose, M. D., Cherniack, N. S. (1982). Effect of aging on the perception of resistive ventilatory loads. American Review of Respiratory Disease, 126, 463-467.

TaCk, M., Altose, M. D., Cherniack, N. S. (1983). Effects of aging on sensation of respiratory force and displacement. Journal of Applied Physiology, 55, 1433-1440.

Teghtsoonian, R. (1971). On the exponents in Stevens' law and the constant in Ekman's law. Psychological Review, 78, 71-80.

Teghtsoonian, R., \& Teghtsoonian, M. (1982). Perceived effort in sniffing: The effects of sniff pressure and resistance. Perception \& Psychophysics, 31, 324-329.

WARD, M. E., \& Stubbing, D. G. (1985). Effect of chronic lung disease on the perception of added inspiratory loads. American Review of Respiratory Disease, 132, 652-656.

ZeCHMAN, F. W., \& WILEY, R. L. (1986). Afferent inputs to breathing: Respiratory sensation. In A. P. Fishman (Ed.), Handbook of physiology (Sec. 3, Vol. 2, Pt. 1, pp. 449-474). Bethesda, MD: American Physiological Society.

(Manuscript received December 18, 1986; revision accepted for publication March 14, 1988.) 